\title{
General Decay Of A Nonlinear Viscoelastic Wave: Equation With Boundary Dissipation
}

\section{Déficience D’une Onde Viscoélastique Non Linéaire : Équation Avec Dissipation Aux Limites}

\author{
Amel Boudiaf and Salah Drabla \\ Department of Mathematics, Faculty of Science, University of setif, Algeria \\ amel.boudiaf@univ-setif.dz,drabla_s@univ-setif.dz
}

ABSTRACT. In this work we establish a general decay rate for a nonlinear viscoelastic wave equation with boundary dissipation where the relaxation function satisfies $g^{\prime}(t) \leq-\xi(t) g^{p}(t), t \geq 0,1 \leq p \leq \frac{3}{2}$. This work generalizes and improves earlier results in the literature.

2010 Mathematics Subject Classification. 35B37, 35L55, 74D05, 93D15, 93D20.

KEYWORDS. Viscoelastic, General decay, Relaxation function, Dissipation, Wave equation.

\section{Introduction}

It is well known that viscoelastic materials have memory effects, which is due to the mechanical response influenced by the history of the materials themselves. As these materials have a wide application in the natural sciences, their dynamics are interesting and of great importance. From the mathematical point of view, their memory effects are modeled by integrodifferential equations. Hence, questions related to the behavior of the solutions for the PDE system have attracted considerable attention in recent years.

For example, Cavalcanti et al [6] considered the following problem

$$
\begin{array}{cc}
u_{t t}-\triangle u+\int_{0}^{t} g(t-s) \triangle u(s) d s+a(x) u_{t}+|u|^{\gamma} u=0, & \text { in } \Omega \times(0, \infty) \\
u(x, t)=0, & x \in \partial \Omega, t \geq 0 \\
u(x, 0)=u_{0}(x), u_{t}(x, 0)=u_{t}(x), & x \in \Omega,
\end{array}
$$

where $\Omega$ is a bounded domain in $\mathbb{R}^{n}(n \geq 1)$ with a smooth boundary, $\gamma>0$, and $a: \Omega \rightarrow \mathbb{R}^{+}$is a function, which may be null on a part of $\Omega$. The authors established an exponential decay estimate under the conditions that $a(x) \geq a_{0}>0$ on $\omega \subset \Omega$, with meas $\omega>0$ and satisfying some geometry conditions and

$$
-\xi_{1} g(t) \leq g^{\prime}(t) \leq-\xi_{2} g(t), \quad t \geq 0 .
$$

Berrimi and Messaoudi [2] improved the result [6] by introducing a new function. They proved an exponential decay result under weaker conditions on both $a$ and $g$. In fact, they allowed the function a to vanish on any part of $\Omega$, and consequently, the geometry condition imposed on a part of boundary is no longer needed. Later, the same authors [3] and Messaoudi [12] extended the result to a situation in which 
a source term is competing with the viscoelastic dissipation. In [7] Cavalcanti and Oquendo considered the following:

$$
\begin{aligned}
& u_{t t}-k_{0} \triangle u(t)+\int_{0}^{t} g(t-s) \operatorname{div}(a(x) \nabla u(s)) d s+b(x) h\left(u_{t}\right)+f(u)=0 \\
& u(x, 0)=u_{0}(x), u_{t}(x, 0)=u_{1}(x) \\
& u(x, t)=0
\end{aligned}
$$

under some conditions on the relaxation function $g$, they improved the result of [6]. Indeed, they proved that the solution of (1.3) decays exponentially to zero when $g$ is decaying exponentially and $h$ is linear and the solution decays polynomially to zero when $g$ is decaying polynomially and $h$ is nonlinear.

On considering the boundary stabilization, Cavalcanti et al. [5] considered the following problem

$$
\begin{cases}u_{t t}-\Delta u+\int_{0}^{t} g(t-s) \triangle u(s) d s=0, & \text { in } \Omega \times(0, \infty) \\ \frac{\partial u}{\partial \nu}-\int_{0}^{t} g(t-s) \frac{\partial u}{\partial \nu}(s) d s+h\left(u_{t}\right)=0, & \text { on } \Gamma_{1} \times(0, \infty) \\ u(x, t)=0, & \text { on } \Gamma_{0} \times(0, \infty) \\ u(x, 0)=u_{0}, u_{t}(x, 0)=u_{1}, & x \in \Omega .\end{cases}
$$

The existence and uniform decay rate results were established under quite restrictive assumptions on damping term $h$ and the kernel function $g$. Later, Cavalcanti et al. [8] generalized this result without imposing a growth condition on $h$ and under a weaker assumption on $g$. Recently, Messaoudi and Mustafa [13] exploited some properties of convex functions [1] and the multiplier method to extend these results. They established an explicit and general decay rate result without imposing any restrictive growth assumption on the damping term $h$ and greatly weakened the assumption on $g$. Also, Li et al [10] have analyzed the global existence and decay estimates for nonlinear viscoelastic wave equation with boundary dissipation. They established uniform decay rate of the energy under suitable conditions on the initial data and the relaxation function $g$. We refer the reader to related works [[7], [9], [11], [15], [18]] . In the present work, we are concerned with

$$
\begin{array}{ll}
u_{t t}-k_{0} \triangle u(t)+\int_{0}^{t} g(t-s) d i v(a(x) \nabla u(s)) d s+b(x) u_{t}=|u|^{\gamma-2} u, & \text { in } \Omega \times(0, \infty) \\
k_{0} \frac{\partial u}{\partial \nu}-\int_{0}^{t} g(t-s)(a(x) \nabla u(s)) . \nu d s+h\left(u_{t}\right)=0, & \text { on } \Gamma_{1} \times(0, \infty) \\
u=0, & \text { on } \Gamma_{0} \times(0, \infty) \\
u(x, 0)=u_{0}, \quad u_{t}(x, 0)=u_{1}, & x \in \Omega .
\end{array}
$$

Where $k_{0}>0$ and $\Omega$ is a bounded domain in $\mathbb{R}^{n}(n \geq 1)$ with a smooth boundary, $\Gamma=\Gamma_{0} \cup \Gamma_{1}$. Here $\Gamma_{0}$ and $\Gamma_{1}$ are closed and disjoint with meas $\left(\Gamma_{0}\right)>0$, and $\nu$ is the unit outward normal to $\Gamma . b: \Omega \rightarrow \mathbb{R}^{+}$is a function, and

$$
\begin{aligned}
& 2<\gamma \leq \frac{2 n}{n-2}, \quad n \geq 3 \\
& \gamma>2, \quad \text { if } n=1,2
\end{aligned}
$$

Our aim in this work is to obtain a more general and explicit energy decay formula, from which the usual exponential and polynomial decay rates are only special cases of our result. In fact, our decay formulae extend and improve some results in the literature. 


\section{Preliminaries}

In this section we prepare some material needed in the proof of our result. According to (1.6) we have the imbedding: $H_{\Gamma_{0}}^{1} \hookrightarrow L^{2(\gamma+1)}(\Omega)$. Let $B>0$ be the optimal constant of Sobolev imbedding which satisfies the following inequality:

$$
\|u\|_{2(\gamma+1)} \leq B\|\nabla u\|_{2}, \quad \forall u \in H_{\Gamma_{0}}^{1}
$$

and we use the trace-Sobolev imbedding: $H_{\Gamma_{0}}^{1} \hookrightarrow L^{k}\left(\Gamma_{1}\right), 1 \leq k<\frac{2(n-1)}{n-2}$. In this case, the imbedding constant is denoted by $B_{1}$, that is

$$
\|u\|_{k, \Gamma_{1}} \leq B_{1}\|\nabla u\|_{2}
$$

Next, we state the assumptions for problem (1.5) as follows.

(A1) $g: \mathbb{R}^{+} \rightarrow \mathbb{R}^{+}$is a nonincreasing differentiable function such that

$$
g(0)>0, \quad k_{0}-\int_{0}^{\infty} g(s) d s=l>0 .
$$

(A2) There exists a nonincreasing differentiable function $\xi: \mathbb{R}_{+} \rightarrow \mathbb{R}_{+}$, with $\xi(0)>0$, satisfying

$$
g^{\prime}(t) \leq-\xi(t) g^{p}(t), \quad \forall t \geq 0,1 \leq p<\frac{3}{2} .
$$

$(A 3) h: \mathbb{R} \rightarrow \mathbb{R}$ is a nondecreasing function with

$$
\begin{aligned}
& h(s) s \geq \alpha|s|^{2}, \quad \forall s \in \mathbb{R}, \\
& |h(s)| \leq \beta|s|, \quad \forall s \in \mathbb{R} .
\end{aligned}
$$

$(A 4) a: \Omega \rightarrow \mathbb{R}$ is a nonnegative functions and $a \in C^{1}(\bar{\Omega})$ such that

$$
\begin{aligned}
a(x) & \geq a_{0}>0, \\
|\nabla a(x)|^{2} & \leq a_{1}^{2}|a(x)|,
\end{aligned}
$$

for some positive constant $a_{1}$.

Remark 2.1. An example of functions satisfying $(A 1)$ and $(A 2)$, respectively, are

- $g(s)=e^{-a s}, a>1$,

- $g(s)=b(1+s)^{-1 /(p-1)}, p>1, b<(2-p) /(p-1)$.

Remark 2.2. Shun and Hsueh [17] considered (1.5) for $p=1$.

Remark 2.3. Condition $p<\frac{3}{2}$ is the consequence of Lemma 2.6 when we take $\sigma=\frac{1}{2}$ to ensure that the term $\int_{0}^{+\infty} \xi(t) g^{\frac{1}{2}}(s) d s$ used in 2.14 must be defined $\left(\int_{0}^{+\infty} \xi(t) g^{\frac{1}{2}}(s) d s<\infty\right)$.

By using the Galerkin method and procedure similar to that of [10] and [16], we have the following local existence result for problem (1.5). 
Theorem 2.4. Let hypotheses (A1)-(A4) hold and (1.6) hold and assume that $u_{0} \in H_{\Gamma_{0}}^{1} \cap H^{2}(\Omega)$, $u_{1} \in H_{\Gamma_{0}}^{1}$. Then there exists a strong solution u of (1.5) satisfying

$$
\begin{aligned}
u & \in L^{\infty}\left([0, T) ; H_{\Gamma_{0}}^{1} \cap H^{2}(\Omega)\right) \\
u_{t} & \in L^{\infty}\left([0, T) ; H_{\Gamma_{0}}^{1}\right) \\
u_{t t} & \in L^{\infty}\left([0, T) ; L^{2}(\Omega)\right),
\end{aligned}
$$

for some $T>0$.

We introduce the following functionals

$$
\begin{aligned}
& J(t)=\frac{1}{2}\left(k_{0}-a(x) \int_{0}^{t} g(s) d s\right)\|\nabla u\|_{2}^{2}+\frac{1}{2}(g \circ \nabla u)(t)-\frac{1}{\gamma}\|u\|_{\gamma}^{\gamma} \\
& E(t)=J(u(t))+\frac{1}{2}\left\|u_{t}\right\|_{2}^{2}, \text { for } t \in[0, T) \\
& I(t)=I(u(t))=\left(k_{0}-\int_{0}^{t} g(s) d s\right)\|\nabla u\|_{2}^{2}+(g \circ \nabla u)(t)-\|u\|_{\gamma}^{\gamma},
\end{aligned}
$$

where

$$
(g \circ v)(t)=\int_{0}^{t} g(t-s)\|v(t)-v(s)\|_{2}^{2} d s,
$$

and $E(t)$ is the energy functional.

A direct differentiation, using (1.5), leads to

$$
E^{\prime}(t)=\frac{1}{2}\left(g^{\prime} \circ \nabla u\right)(t)-\frac{1}{2} \int_{\Omega} a(x) g(t)|\nabla u(t)|^{2} d x-\int_{\Omega} b(x)\left|u_{t}(t)\right|^{2} d x \leq 0 .
$$

We start with the following crucial lemma which will be used in the proof of our result.

Lemma 2.5. [10]For any $u \in C^{1}\left(0, T ; H^{1}(\Omega)\right)$, we have

$$
\begin{aligned}
\int_{\Omega} \int_{0}^{t} g(t-s) \nabla u(s) \nabla u_{t} d s d x= & -\frac{1}{2} \int_{\Omega} g(t)|\nabla u(t)|^{2} d x+\frac{1}{2}\left(g^{\prime} \circ \nabla u\right)(t) \\
& -\frac{1}{2} \frac{d}{d t}\left[(g \circ \nabla u)(t)-\int_{\Omega} \int_{0}^{t} g(s) d s|\nabla u(t)|^{2} d x\right] .
\end{aligned}
$$

Lemma 2.6. Assume that $g$ satisfies $(A 1)$ and $(A 2)$ then

$$
\int_{0}^{+\infty} \xi(t) g^{1-\sigma}(t) d t<+\infty, \quad \forall \sigma<2-p
$$


Proof. Recalling $(A 1)$ and $(A 2)$, we easily see that

$$
\xi(t) g^{1-\sigma}(t)=\xi(t) g^{1-\sigma}(t) g^{p}(t) g^{-p}(t) \leq-g^{\prime}(t) g^{1-\sigma-p}(t) .
$$

Integration then gives

$$
\left.\int_{0}^{+\infty} \xi(t) g^{1-\sigma}(t) d t \leq-\int_{0}^{+\infty} g^{\prime}(t) g^{1-\sigma-p}(t)=-\frac{g^{2-p-\sigma}(t)}{2-p-\sigma}\right]_{0}^{+\infty}<+\infty,
$$

since $\sigma<2-p$.

The next lemma and corollary are crucial for the proof of our main result.

Lemma 2.7. [14] Assume that $g$ satisfies (A1) and (A2), and $u$ is the solution of (1.5) then, for $0<\sigma<$ 1 , we have

$$
(g \circ \nabla u)(t) \leq C\left[\left(\int_{0}^{+\infty} g^{1-\sigma}(t) d t\right) E(0)\right]^{\frac{p-1}{p-1+\sigma}}\left(g^{p} \circ \nabla u\right)^{\frac{\sigma}{p-1+\sigma}}(t) .
$$

By taking $\sigma=\frac{1}{2}$, we get

$$
(g \circ \nabla u)(t) \leq C\left[\int_{0}^{t} g^{\frac{1}{2}}(s) d s\right]^{\frac{2 p-2}{2 p-1}}\left(g^{p} \circ \nabla u\right)^{\frac{1}{2 p-1}}(t) .
$$

Corollary 2.8. Assume that $g$ satisfies $(A 1)$ and $(A 2)$, and $u$ is the solution of $(1.5)$ then

$$
\xi(t)(g \circ \nabla u)(t) \leq C\left[-E^{\prime}(t)\right]^{\frac{1}{2 p-1}} .
$$

Proof. Multiply both sides of (2.12) by $\xi(t)$ and recall Lemma 2.6 and (2.10) to get

$$
\begin{aligned}
\xi(t)(g \circ \nabla u)(t) & \leq C \xi(t)^{\frac{2 p-2}{2 p-1}}(t)\left[\int_{0}^{t} g^{\frac{1}{2}}(s) d s\right]^{\frac{2 p-2}{2 p-1}} \xi^{\frac{1}{2 p-1}}(t)\left(g^{p} \circ \nabla u\right)^{\frac{1}{2 p-1}}(t) \\
& \leq C\left[\int_{0}^{t} \xi(t) g^{\frac{1}{2}}(s) d s\right]^{\frac{2 p-2}{2 p-1}}\left(\xi g^{p} \circ \nabla u\right)^{\frac{1}{2 p-1}}(t) \\
& \leq C\left[\int_{0}^{+\infty} \xi(s) g^{\frac{1}{2}}(s) d s\right]^{\frac{2 p-2}{2 p-1}}\left(-g^{\prime} \circ \nabla u\right)^{\frac{1}{2 p-1}}(t) \leq C\left[-E^{\prime}(t)\right]^{\frac{1}{2 p-1}} .
\end{aligned}
$$

We also recall well-known Jensen's inequality which will be of essential use in obtaining our result. 
If $G$ is a convex function on $[a, b],(-G$ is convex $), f: \Omega \rightarrow[a, b]$ and $h$ are integrable functions on $\Omega$, with $h(x) \geq 0$ and $\int_{\Omega} h(x) d x=k>0$, then Jensen's inequality states that

$$
\frac{1}{k} \int_{\Omega} G[f(x)] h(x) d x \leq G\left[\frac{1}{k} \int_{\Omega} f(x) h(x) d x\right] .
$$

For the special case $G(y)=y^{\frac{1}{q}}, y \geq 0, p>1$, we have

$$
\frac{1}{k} \int_{\Omega}[f(x)]^{\frac{1}{q}} h(x) d x \leq\left[\frac{1}{k} \int_{\Omega} f(x) h(x) d x\right]^{\frac{1}{q}} .
$$

\section{Global existence}

We state and prove the global existence result.

Lemma 3.1. Suppose that (A1), (A3) and (1.6)hold. Assume further that $u_{0} \in H_{\Gamma_{0}}^{1}, u_{1} \in L^{2}(\Omega)$, such that

$$
\beta=\frac{B^{\gamma}}{l}\left(\frac{2 \gamma}{(\gamma-2) l} E(0)\right)^{(\gamma-2) / 2}<1,
$$

and $I\left(u_{0}\right)>0$, then $I(u(t))>0, \forall t>0$, where $B$ is the best Poincare constant, and $E(0)=$ $E\left(u_{0}, u_{1}\right)$.

Proof. Since $I\left(u_{0}\right)>0$, then there exists (by continuity) $T_{m}<T$ such that

$$
I(u(t)) \geq 0, \forall t \in\left[0, T_{m}\right],
$$

this gives

$$
\begin{aligned}
J(t) & =\frac{1}{2}\left(k_{0}-\int_{0}^{t} g(s) d s\right)\|\nabla u\|_{2}^{2}+\frac{1}{2}(g \circ \nabla u)(t)-\frac{1}{\gamma}\|u\|_{\gamma}^{\gamma} \\
& =\left(\frac{\gamma-2}{2 \gamma}\right)\left(\left(k_{0}-\int_{0}^{t} g(s) d s\right)\|\nabla u(t)\|_{2}^{2}+(g \circ \nabla u)(t)\right)+\frac{1}{\gamma} I(t) \\
& \geq\left(\frac{\gamma-2}{2 \gamma}\right)\left(\left(k_{0}-\int_{0}^{t} g(s) d s\right)\|\nabla u(t)\|_{2}^{2}+(g \circ \nabla u)(t)\right) .
\end{aligned}
$$

By using $(A 1),(2.8),(3.1)$ and (3.2) we have

$$
\begin{aligned}
l\|\nabla u\|_{2}^{2} & \leq\left(k_{0}-\int_{0}^{t} g(s) d s\right)\|\nabla u(t)\|_{2}^{2} \leq\left(\frac{2 \gamma}{\gamma-2}\right) J(t) \\
& \leq\left(\frac{2 \gamma}{\gamma-2}\right) E(t) \leq\left(\frac{2 \gamma}{\gamma-2}\right) E(0), \quad \forall t \in\left[0, T_{m}\right]
\end{aligned}
$$


we then exploit $(A 1),(2.1),(3.1)$ and $(3.3)$ to obtain

$$
\begin{aligned}
\|u\|_{\gamma}^{\gamma} & \leq B^{\gamma}\|\nabla u(t)\|_{2}^{\gamma} \\
& \leq \frac{B^{\gamma}}{l}\|\nabla u(t)\|_{2}^{\gamma-2} l\|\nabla u(t)\|_{2}^{2} \leq \beta l\|\nabla u(t)\|_{2}^{2} \\
& \leq \beta\left(k_{0}-\int_{0}^{t} g(s) d s\right)\|\nabla u(t)\|_{2}^{2} \\
& <\left(k_{0}-\int_{0}^{t} g(s) d s\right)\|\nabla u(t)\|_{2}^{2}, \forall t \in\left[0, T_{m}\right]
\end{aligned}
$$

Therefore

$$
I(t)=\left(k_{0}-\int_{0}^{t} g(s) d s\right)\|\nabla u\|_{2}^{2}+(g \circ \nabla u)(t)-\|u\|_{\gamma}^{\gamma}>0,
$$

for all $t \in\left[0, T_{m}\right]$. By repeating this procedure and using the fact that

$$
\lim _{t \rightarrow T_{m}} \frac{B^{\gamma}}{l}\left(\frac{2 \gamma}{(\gamma-2) l} E(0)\right)^{(\gamma-2) / 2} \leq \beta<1
$$

$T_{m}$ is extended to $T$.

Theorem 3.2. Suppose that $(A 1),(A 2)$ and (1.6)hold. If $\left(u_{0}, u_{1}\right) \in H_{\Gamma_{0}}^{1}(\Omega) \times L^{2}(\Omega)$ and satisfies (3.1). Then the solution is global and bounded.

Proof. It suffices to show that

$$
\|\nabla u(t)\|_{2}^{2}+\left\|u_{t}(t)\right\|_{2}^{2}
$$

is bounded independently of $t$. To achieve this, we use (2.8), (2.10) and (3.2) to get

$$
\begin{aligned}
E(0) & \geq E(t)=\frac{1}{2}\left\|u_{t}\right\|_{2}^{2}+J(u(t)) \\
& \geq\left(\frac{\gamma-2}{2 \gamma}\right)\left(l\|\nabla u(t)\|_{2}^{2}+(g \circ \nabla u)(t)\right)+\frac{1}{2}\left\|u_{t}(t)\right\|_{2}^{2}+\frac{1}{\gamma} I(t) \\
& \geq\left(\frac{\gamma-2}{2 \gamma}\right) l\|\nabla u(t)\|_{2}^{2}+\frac{1}{2}\left\|u_{t}(t)\right\|_{2}^{2}
\end{aligned}
$$

since $I(t)$ and $(g \circ \nabla u)(t)$ are positive. Therefore

$$
\|\nabla u(t)\|_{2}^{2}+\left\|u_{t}(t)\right\|_{2}^{2} \leq C E(0)
$$

where $c$ is a positive constant, which depends only on $\gamma$ and $l$.

\section{Decay of solutions}

In this section we state and prove the main result of our work. First, we define some functionals. Let

$$
\mathcal{F}(t)=E(t)+\varepsilon_{1} \Phi(t)+\varepsilon_{2} \Psi(t),
$$


where

$$
\begin{aligned}
& \Phi(t)=\int_{\Omega} u \cdot u_{t} d x \\
& \Psi(t)=\int_{\Omega} a(x) u_{t} \int_{0}^{t} g(t-s)(u(s)-u(t)) d s d x,
\end{aligned}
$$

and $\varepsilon_{1}, \varepsilon_{2}$ are some positive constants to be specified later.

Lemma 4.1. There exist two positive constants $\beta_{1}$ and $\beta_{2}$ such that the relation

$$
\beta_{1} E(t) \leq \mathcal{F}(t) \leq \beta_{2} E(t)
$$

holds, for $\varepsilon_{1}, \varepsilon_{2}>0$ small enough.

Proof. By Hölder's inequality, Young's inequality, (2.1) and (2.7), we deduce that

$$
\begin{aligned}
|\Phi(t)| & \leq \frac{1}{2}\left\|u_{t}\right\|_{2}^{2}+\frac{B^{2}}{2}\|\nabla u\|_{2}^{2} \\
|\Psi(t)| & \leq \frac{1}{2}\left\|u_{t}\right\|_{2}^{2}+\frac{1}{2} \int_{\Omega}\left(\int_{0}^{t} g(t-s)(u(t)-u(s)) d s\right)^{2} d x \\
& \leq \frac{1}{2}\left\|u_{t}\right\|_{2}^{2}+\frac{1}{2} \int_{0}^{t} g(s) d s \int_{\Omega} \int_{0}^{t} g(t-s)|u(t)-u(s)|^{2} d s d x \\
& \leq \frac{1}{2}\left\|u_{t}\right\|_{2}^{2}+\frac{\left(k_{0}-l\right)\|a\|_{\infty} B^{2}}{2 a_{0}}(g \circ \nabla u)(t) .
\end{aligned}
$$

Hence, taking (4.1), (4.5) and (4.6) into account, we have

$$
\begin{aligned}
\mathcal{F}(t) & =E(t)+\varepsilon_{1} \Phi(t)+\varepsilon_{2} \Psi(t) \\
& \leq E(t)+c_{1}\left\|u_{t}\right\|_{2}^{2}+c_{2}\|\nabla u\|_{2}^{2}+c_{3}(g \circ \nabla u)(t), \\
\mathcal{F}(t) & \geq E(t)-c_{4}\left(\left\|u_{t}\right\|_{2}^{2}+\|\nabla u\|_{2}^{2}+(g \circ \nabla u)(t)\right),
\end{aligned}
$$

where $c_{1}=\left(\varepsilon_{1}+\varepsilon_{2}\right) / 2, c_{2}=\varepsilon_{1} B^{2} / 2, c_{3}=\left(k_{0}-l\right)\|a\|_{\infty} B^{2} \varepsilon_{2} / 2 a_{0}$, and $c_{4}=\max \left(c_{1}, c_{2}, c_{3}\right)$. Thus, selecting $\varepsilon_{1}, \varepsilon_{2}$ small enough, there exist two positive constants $\beta_{1}$ and $\beta_{2}$ such that

$$
\beta_{1} E(t) \leq \mathcal{F}(t) \leq \beta_{2} E(t)
$$

Lemma 4.2. Assume that $(A 1)-(A 4)$, hold, then the functional

$$
\Phi(t)=\int_{\Omega} u . u_{t} d x,
$$


satisfies, along the solution of (1.5),

$$
\begin{aligned}
\Phi^{\prime}(t) \leq & -\frac{1}{4}\|\nabla u\|_{2}^{2}+\left\|u_{t}\right\|_{2}^{2}+\frac{\left(k_{0}-l\right)}{2 l}(g \circ \nabla u)(t)+\frac{2 \beta^{2} B_{*}^{2}}{l} \int_{\Gamma_{1}} u_{t}^{2} d \Gamma \\
& +\frac{2 B^{2}\|b\|_{\infty}}{l} \int_{\Omega} b(x) u_{t}^{2} d x+\|u\|_{\gamma}^{\gamma} .
\end{aligned}
$$

Proof. We estimate the derivative of $\Phi(t)$. From (4.2) and using (1.5), we have

$$
\begin{aligned}
\Phi^{\prime}(t)= & \left\|u_{t}\right\|_{2}^{2}-k_{0}\|\nabla u\|_{2}^{2}+\int_{\Omega} \nabla u(t) a(x) \int_{0}^{t} g(t-s) \nabla u(s) d s d x \\
& -\int_{\Gamma_{1}} h\left(u_{t}\right) u d \Gamma-\int_{\Omega} b(x) u_{t} u d x+\|u\|_{\gamma}^{\gamma} .
\end{aligned}
$$

The third, the fourth, and the fifth terms on the right-hand side of (4.11) can be estimated as follows. From Hölder's inequality, Young's inequality, and (2.11), for $\eta>0$, we have

$$
\begin{aligned}
& \int_{\Omega} \nabla u(t) a(x) \int_{0}^{t} g(t-s) \nabla u(s) d s d x \\
\leq & \frac{k_{0}}{2} \int_{\Omega}|\nabla u|^{2} d x+\frac{1}{2 k_{0}} \int_{\Omega} a(x)\left(\int_{0}^{t} g(t-s)(\nabla u(s)-\nabla u(t)+\nabla u(t)) d s\right)^{2} d x \\
\leq & {\left[\frac{k_{0}}{2}+\frac{1}{2 k_{0}}(1+\eta)\left(k_{0}-l\right)^{2}\right]\|\nabla u\|_{2}^{2}+\frac{1}{2 k_{0}}\left(1+\frac{1}{\eta}\right)\left(k_{0}-l\right)(g \circ \nabla u)(t) . }
\end{aligned}
$$

Employing Hölder's inequality, Young's inequality, $(A 1)$, and $(2.1)$, for $\delta_{1}, \delta_{2}>0$, we see that

$$
\begin{aligned}
& \left|\int_{\Gamma_{1}} h\left(u_{t}\right) u d \Gamma\right| \leq \delta_{1} B_{*}^{2}\|\nabla u\|_{2}^{2}+\frac{\beta^{2}}{4 \delta_{1}} \int_{\Gamma_{1}} u_{t}^{2} d \Gamma . \\
& \int_{\Omega} b(x) u_{t} u d x \leq B^{2}\|b\|_{\infty} \delta_{2}\|\nabla u\|_{2}^{2}+\frac{1}{4 \delta_{2}} \int_{\Omega} b(x) u_{t}^{2} d x .
\end{aligned}
$$

A substitution of (4.12) - (4.13) into (4.11) yields, we arrive at

$$
\begin{aligned}
\Phi^{\prime}(t) \leq & \left\|u_{t}\right\|_{2}^{2}-\left(\frac{k_{0}}{2}-\frac{1}{2 k_{0}}(1+\eta)\left(k_{0}-l\right)^{2}-\delta_{1} B_{*}^{2}-B^{2}\|b\|_{\infty} \delta_{2}\right)\|\nabla u\|_{2}^{2} \\
& +\frac{1}{2 k_{0}}\left(1+\frac{1}{\eta}\right)\left(k_{0}-l\right)(g \circ \nabla u)(t)+\frac{\beta^{2}}{4 \delta_{1}} \int_{\Gamma_{1}} u_{t}^{2} d \Gamma \\
& +\frac{1}{4 \delta_{2}} \int_{\Omega} b(x) u_{t}^{2} d x+\|u\|_{\gamma}^{\gamma} .
\end{aligned}
$$


Letting $\eta=l /\left(k_{0}-l\right)>0, \delta_{1}=l / 8 B_{*}^{2}$, and $\delta_{2}=l / 8 \beta^{2}\|b\|_{\infty}$ in the above inequality, we obtain

$$
\begin{aligned}
\Phi^{\prime}(t) \leq & -\frac{1}{4}\|\nabla u\|_{2}^{2}+\left\|u_{t}\right\|_{2}^{2}+\frac{\left(k_{0}-l\right)}{2 l}(g \circ \nabla u)(t)+\frac{2 \beta^{2} B_{*}^{2}}{l} \int_{\Gamma_{1}} u_{t}^{2} d \Gamma \\
& +\frac{2 B^{2}\|b\|_{\infty}}{l} \int_{\Omega} b(x) u_{t}^{2} d x+\|u\|_{\gamma}^{\gamma} .
\end{aligned}
$$

Then (4.10) is established.

Lemma 4.3. Assume that (A1)-(A4) hold, then the functional

$$
\Psi(t)=\int_{\Omega} a(x) u_{t} \int_{0}^{t} g(t-s)(u(s)-u(t)) d s d x,
$$

satisfies for some positive constants $c_{5}, c_{6}$,

$$
\begin{aligned}
\Psi^{\prime}(t) \leq & -\left(a_{0} \int_{0}^{t} g(s) d s-\delta\right)\left\|u_{t}\right\|_{2}^{2}+\delta c_{5}\|\nabla u\|_{2}^{2}+c_{6}(g \circ \nabla u)(t)+\delta\|b\|_{\infty} \int_{\Omega} b(x) u_{t}^{2} d x \\
& -\frac{g(0)\|a\|_{\infty}^{2} B^{2}}{4 a_{0} \delta}\left(g^{\prime} \circ \nabla u\right)(t)+\delta \beta^{2} \int_{\Gamma_{1}} u_{t}^{2} d \Gamma
\end{aligned}
$$

Proof. Taking the derivative of $\Psi(t)$, and using (1.5) to obtain

$$
\begin{aligned}
\Psi^{\prime}(t)= & k_{0} \int_{\Omega} a(x) \nabla u(t)\left(\int_{0}^{t} g(t-s)(\nabla u(t)-\nabla u(s)) d s\right) d x \\
& +k_{0} \int_{\Omega} \nabla a(x) \cdot \nabla u(t) \int_{0}^{t} g(t-s)(u(t)-u(s)) d s d x \\
& -\int_{\Omega}\left(\int_{0}^{t} g(t-s) a(x) \nabla u(s) \nabla a(x) d s\right)\left(\int_{0}^{t} g(t-s)(u(t)-u(s)) d s\right) d x \\
& -\int_{\Omega} a(x)\left(\int_{0}^{t} g(t-s) a(x) \nabla u(s) d s\right)\left(\int_{0}^{t} g(t-s)(\nabla u(t)-\nabla u(s)) d s\right) d x \\
& +\int_{\Omega} a(x) b(x) u_{t} \int_{0}^{t} g(t-s)(u(t)-u(s)) d s d x \\
& +\int_{\Gamma_{1}} a(x) h\left(u_{t}\right) \int_{0}^{t} g(t-s)(u(t)-u(s)) d s d \Gamma \\
& -\int_{\Omega} a(x)|u|^{p-2} u \int_{0}^{t} g(t-s)(u(t)-u(s)) d s d x \\
& -\int_{\Omega} a(x) u_{t} \int_{0}^{\prime} g^{\prime}(t-s)(u(t)-u(s)) d s d x-\left(\int_{0}^{t} g(s) d s\right) \int_{\Omega} a(x) u_{t}^{2} d x . \quad(4.17)
\end{aligned}
$$


Similarly to (4.11), we estimate the right side of (4.17). Using Young's inequality, Hölder's inequality, $(2.11),(A 1),(A 2)$ and $(A 3)$, for $\delta>0$, we have

$$
\begin{aligned}
& \bullet\left|\int_{\Omega} k_{0} \nabla u(t) a(x) \int_{0}^{t} g(t-s)(\nabla u(t)-\nabla u(s)) d s d x\right| \\
\leq & \left.k_{0}^{2} \delta\|\nabla u\|_{2}^{2}+\frac{1}{4 \delta} \int_{\Omega} \int_{0} a(x) \int_{0}^{t} g(t-s)(\nabla u(t)-\nabla u(s)) d s\right)^{2} d x \\
\leq & k_{0}^{2} \delta\|\nabla u\|_{2}^{2}+\frac{\|a\|_{\infty}}{4 \delta} \int_{0}^{t} g(s) d s \int_{\Omega} a(x) \int_{0}^{t} g(t-s)|\nabla u(t)-\nabla u(s)|^{2} d s d x \\
\leq & k_{0}^{2} \delta\|\nabla u\|_{2}^{2}+\frac{k_{0}-l}{4 \delta}(g \circ \nabla u)(t), \\
& \bullet \int_{\Omega} k_{0} \nabla u(t) \cdot \nabla a(x) \int_{0}^{t} g(t-s)(u(t)-u(s)) d s d x \\
\leq & k_{0} a_{1} \int_{\Omega}|\nabla u(t)| \sqrt{a(x)}\left(\int_{0}^{t} g(s) d s\right)^{\frac{1}{2}}\left(\int_{0}^{t} g(t-s)(u(t)-u(s)) d s\right)^{\frac{1}{2}} d x \\
\leq & k_{0}^{2} a_{1}^{2} \delta\|\nabla u\|_{2}^{2}+\frac{\left(k_{0}-l\right) B^{2}}{4 \delta a_{0}}(g \circ \nabla u)(t) .
\end{aligned}
$$

Again, exploiting (2.3) , (2.7), Young's inequality and Hölder's inequality, we obtain

$$
\begin{aligned}
& \bullet\left|\int_{\Omega}\left(\int_{0}^{t} g(t-s) a(x) \nabla u(s) \nabla a(x) d s\right)\left(\int_{0}^{t} g(t-s)(u(t)-u(s)) d s\right) d x\right| \\
\leq & a_{1}^{2} \delta \int_{\Omega} a^{2}(x)\left(\int_{0}^{t} g(t-s)|\nabla u(s)| d s\right) d x \\
& +\frac{1}{4 \delta} \int_{\Omega} a(x)\left(\int_{0}^{t} g(t-s)(u(t)-u(s)) d s\right)^{2} d x \\
\leq & 2 a_{1}^{2} \delta\left(k_{0}-l\right)^{2}\|\nabla u\|_{2}^{2}+\left(2 a_{1}^{2} \delta\left(k_{0}-l\right)+\frac{\left(k_{0}-l\right) B^{2}}{4 \delta a_{0}}\right)(g \circ \nabla u)(t), \\
& \bullet \int_{\Omega} a(x)\left(\int_{0}^{t} g(t-s) a(x) \nabla u(s) d s\right)\left(\int_{0}^{t} g(t-s)(\nabla u(t)-\nabla u(s)) d s\right) d x \\
\leq & 2 \delta\left(k_{0}-l\right)^{2}\|\nabla u\|_{2}^{2}+\left(2 \delta+\frac{1}{4 \delta}\right)\left(k_{0}-l\right)(g \circ \nabla u)(t),
\end{aligned}
$$




$$
\begin{aligned}
& \bullet\left|\int_{\Omega} a(x) b(x) u_{t} \int_{0}^{t} g(t-s)(u(t)-u(s)) d s d x\right| \\
\leq & \delta\|b\|_{\infty} \int_{\Omega} b(x) u_{t}^{2} d x+\frac{\left(k_{0}-l\right)\|a\|_{\infty} B^{2}}{4 \delta a_{0}}(g \circ \nabla u)(t) .
\end{aligned}
$$

Using Young's inequality, Hölder's inequality, (2.1), (2.5), and (2.6), the sixth term on the right hand side of (4.17) can be estimated as

$$
\begin{aligned}
\bullet & \left|\int_{\Gamma_{1}} a(x) h\left(u_{t}\right) \int_{0}^{t} g(t-s)(u(t)-u(s)) d s d \Gamma\right| \\
\leq & \delta \beta^{2} \int_{\Gamma_{1}} u_{t}^{2} d \Gamma+\frac{\left(k_{0}-l\right)\|a\|_{\infty} B^{2}}{4 a_{0} \delta}(g \circ \nabla u)(t) .
\end{aligned}
$$

As for the seventh and the eighth terms on the right-hand side of (4.17), using Hölder's inequality, Young's inequality,(2.1), (2.3), and (3.4), we obtain

$$
\begin{aligned}
& \left.\left|\int_{\Omega} a(x) u\right| u\right|^{\gamma-2} \int_{0}^{t} g(t-s)(u(t)-u(s)) d s d x \mid \\
\leq & \delta\|u\|_{2(\gamma-1)}^{2(\gamma-1)}+\frac{\left(k_{0}-l\right)\|a\|_{\infty} B^{2}}{4 a_{0} \delta}(g \circ \nabla u)(t) \\
\leq & \delta B^{2(\gamma-1)}\left(\frac{2 \gamma E(0)}{l(\gamma-2)}\right)^{\gamma-2}\|\nabla u\|_{2}^{2}+\frac{\left(k_{0}-l\right)\|a\|_{\infty} B^{2}}{4 \delta a_{0}}(g \circ \nabla u)(t), \\
\leq & \delta\left\|u_{t}\right\|_{2}^{2}-\frac{\int_{0} a(0)\|a\|_{\infty}^{2} B_{*}^{2}}{4 \delta a_{0}}\left(g^{\prime} \circ \nabla u\right)(t) .
\end{aligned}
$$

Combining these estimates (4.18)-(4.25), (4.17) becomes

$$
\begin{aligned}
\Psi^{\prime}(t) \leq & -\left(a_{0} \int_{0}^{t} g(s) d s-\delta\right)\left\|u_{t}\right\|_{2}^{2}+\delta c_{5}\|\nabla u\|_{2}^{2}+c_{6}(g \circ \nabla u)(t) \\
& -\frac{g(0)\|a\|_{\infty}^{2} B^{2}}{4 \delta a_{0}}\left(g^{\prime} \circ \nabla u\right)(t)+\delta\|b\|_{\infty} \int_{\Omega} b(x) u_{t}^{2} d x+\delta \beta^{2} \int_{\Gamma_{1}} u_{t}^{2} d \Gamma,
\end{aligned}
$$

where

$$
c_{5}=k_{0}^{2}\left(a_{1}^{2}+1\right)+2\left(a_{1}^{2}+1\right)\left(k_{0}-l\right)^{2}+B^{2(\gamma-1)}\left(\frac{2 \gamma E(0)}{l(\gamma-2)}\right)^{\gamma-2}>0
$$




$$
c_{6}=\left(k_{0}-l\right)\left(\frac{B^{2}}{2 \delta a_{0}}+2 \delta a_{1}^{2}+\left(2 \delta+\frac{1}{2 \delta}\right)+\|a\|_{\infty} \frac{B_{*}^{2}+3 B^{2}}{4 \delta a_{0}}\right)>0,
$$

then (4.16) is established.

We are ready to state and prove our main result.

Theorem 4.4. Let $\left(u_{0}, u_{1}\right) \in\left(H_{\Gamma_{0}}^{1} \times L^{2}(\Omega)\right)$ be given . Assume that $(A 1)-(A 4)$ are satisfied. Then, for any $t_{0}>0$, there exist two positive constants $K$, and $\lambda$ such that the solution of (1.5) satisfies, for all $t \geq t_{0}$

$$
\begin{aligned}
& E(t) \leq K e^{-\lambda \int_{t_{0}}^{t} \xi(s) d s}, \quad \text { if } p=1 . \\
& E(t) \leq K\left[\frac{1}{1+\int_{t_{0}}^{t} \xi^{2 p-1}(s) d s}\right]^{\frac{1}{2 p-2}}, p>1 .
\end{aligned}
$$

Moreover, if

$$
\int_{0}^{+\infty}\left[\frac{1}{t \xi^{2 p-1}(t)+1}\right]^{\frac{1}{2 p-2}} d t<+\infty, \quad 1<p<\frac{3}{2}
$$

then

$$
E(t) \leq K\left[\frac{1}{1+\int_{t_{0}}^{t} \xi^{p}(s) d s}\right]^{\frac{1}{p-1}}, \quad p>1 .
$$

Simple calculations show that (4.28) and (4.29) yield

$$
\int_{t_{0}}^{+\infty} E(t) d t<+\infty
$$

Proof. By using (2.10), (4.1), (4.10), and (4.16), we obtain

$$
\begin{aligned}
\mathcal{F}^{\prime}(t)= & E^{\prime}(t)+\varepsilon_{1} \Phi^{\prime}(t)+\varepsilon_{2} \Psi^{\prime}(t) \\
\mathcal{F}^{\prime}(t) \leq & -\left(\varepsilon_{2}\left(a_{0} g_{0}-\delta\right)-\varepsilon_{1}\right)\left\|u_{t}\right\|_{2}^{2}-\left(\frac{\varepsilon_{1} l}{4}-\varepsilon_{2} \delta c_{5}\right)\|\nabla u\|_{2}^{2} \\
& +\left(\varepsilon_{2} c_{6}+\frac{\left(k_{0}-l\right) \varepsilon_{1}}{2 l}\right)(g \circ \nabla u)(t)-\left(1-\frac{2 \varepsilon_{1} B^{2}\|b\|_{\infty}}{l}-\varepsilon_{2} \delta\|b\|_{\infty}\right) \int_{\Omega} b(x) u_{t}^{2} d x \\
& -\left(\alpha-\frac{2 B_{*}^{2} \varepsilon_{1} \beta^{2}}{l}-\varepsilon_{2} \delta \beta^{2}\right) \int_{\Gamma_{1}}\left|u_{t}\right|^{2} d \Gamma \\
& -\left(\frac{1}{2}-\varepsilon_{2} \frac{g(0)\|a\|_{\infty}^{2} B^{2}}{4 a_{0} \delta}\right)\left(-g^{\prime} \circ \nabla u\right)(t)+\varepsilon_{1}\|u\|_{\gamma}^{\gamma} d x, \quad \forall t \geq t_{0},
\end{aligned}
$$

we have used the fact that for any $t_{0}>0$,

$$
\int_{0}^{t} g(s) d s \geq \int_{0}^{t_{0}} g(s) d s=g_{0}, \quad \forall t \geq t_{0}
$$


because $g$ is positive and continuous with $g(0)>0$. At this point, we choose $\delta$ small enough so that

$$
\frac{4 \delta c_{5}}{l}<\frac{a_{0} g_{0}}{2}<a_{0} g_{0}-\delta
$$

Whence $\delta$ is fixed, the choice of any two positive constants $\varepsilon_{1}$ and $\varepsilon_{2}$ satisfying

$$
\frac{4 \delta \varepsilon_{2} c_{5}}{l}<\varepsilon_{1}<\frac{a_{0} g_{0}}{2} \varepsilon_{2}
$$

will make

$$
k_{1}=\frac{\varepsilon_{1} l}{4}-\varepsilon_{2} \delta c_{5}>0
$$

and

$$
k_{2}=\varepsilon_{2}\left(a_{0} g_{0}-\delta\right)-\varepsilon_{1}>0
$$

Then, we choose $\delta, \varepsilon_{1}$ and $\varepsilon_{2}$ small that (4.9) and (4.33) remain valid, further

$$
\begin{aligned}
& k_{3}=1-\frac{2 \varepsilon_{1} B^{2}\|b\|_{\infty}}{l}-\varepsilon_{2} \delta\|b\|_{\infty}>0, \\
& k_{4}=\alpha-\frac{2 B_{*}^{2} \varepsilon_{1} \beta^{2}}{l}-\varepsilon_{2} \delta \beta^{2}>0, \\
& k_{5}=\frac{1}{2}-\varepsilon_{2} \frac{g(0)\|a\|_{\infty}^{2} B^{2}}{4 a_{0} \delta}>0 .
\end{aligned}
$$

Hence, for all $t_{0}>0$, we arrive at

$$
\begin{aligned}
\mathcal{F}^{\prime}(t) \leq & -k_{1}\|\nabla u\|_{2}^{2}-k_{2}\left\|u_{t}\right\|_{2}^{2}+c_{7}(g \circ \nabla u)(t)+c_{8}\left(g^{\prime} \circ \nabla u\right)(t) \\
& -k_{3} \int_{\Omega} b(x) u_{t}^{2} d x-k_{4} \int_{\Gamma_{1}}\left|u_{t}\right|^{2} d \Gamma+\varepsilon_{1}\|u\|_{\gamma}^{\gamma},
\end{aligned}
$$

which yields (if needed, one can choose $\varepsilon_{1}$ sufficiently small)

$$
\mathcal{F}^{\prime}(t) \leq-m E(t)+C(g \circ \nabla u)(t)
$$

where $c_{i}, i=7,8, m, C$ are some positive constants.

Multiplying (4.41) by $\xi(t)$ gives

$$
\xi(t) \mathcal{F}^{\prime}(t) \leq-m \xi(t) E(t)+C \xi(t)(g \circ \nabla u)(t), \forall t \geq t_{0}
$$

It follows from $(4.41),(2.3)$, and (2.4) that

Case of $p=1$. Recalling (2.4) and (2.10), we obtain, from (4.42), for all $t \geq t_{0}$

$$
\begin{aligned}
\xi(t) \mathcal{F}^{\prime}(t) & \leq-m \xi(t) E(t)+C(\xi g \circ \nabla u)(t) \leq-m \xi(t) E(t)-C\left(g^{\prime} \circ \nabla u\right)(t) \\
& \leq-m \xi(t) E(t)-C E^{\prime}(t)
\end{aligned}
$$

which leads to

$$
(\xi \mathcal{F}+C E)^{\prime}(t) \leq-m \xi(t) E(t), \quad \forall t \geq t_{0} .
$$


Let $L(t):=\xi(t) \mathcal{F}(t)+C E(t)$, then clearly $L \sim E$ and we have, for some $m_{1}>0$,

$$
L^{\prime}(t) \leq-m_{1} \xi(t) L(t), \forall t \geq t_{0}
$$

By a simple integration, we arrive at

$$
L(t) \leq C e^{-m_{1} \int_{t_{0}}^{t} \xi(s) d s}, \quad \forall t \geq t_{0}
$$

Hence (4.27) by virtue of $L \sim E$.

Case of $p>1$. To establish (4.28), we again consider (4.42) and use Corollary 2.8 to get

$$
\xi(t) \mathcal{F}^{\prime}(t) \leq-m \xi(t) E(t)+C\left[-E^{\prime}(t)\right]^{\frac{1}{2 p-1}}, \quad \forall t \geq t_{0}
$$

Multiplication of the last inequality by $\xi^{\alpha} E^{\alpha}(t)$, where $\alpha=2 p-2$, gives

$$
\xi^{\alpha+1}(t) E^{\alpha}(t) \mathcal{F}^{\prime}(t) \leq-m \xi^{\alpha+1}(t) E^{\alpha+1}(t)+C(\xi E)^{\alpha}(t)\left[-E^{\prime}(t)\right]^{\frac{1}{\alpha+1}}, \quad \forall t \geq t_{0} .
$$

Use of Young's inequality, with $q=\alpha+1$ and $q^{*}=\frac{\alpha+1}{\alpha}$, yields

$$
\begin{aligned}
\xi^{\alpha+1}(t) E^{\alpha}(t) \mathcal{F}^{\prime}(t) \leq & -m \xi^{\alpha+1}(t) E^{\alpha+1}(t) \\
& +C\left[\varepsilon \xi^{\alpha+1}(t) E^{\alpha+1}(t)-C_{\varepsilon} E^{\prime}(t)\right] \\
= & -(m-\varepsilon C) \xi^{\alpha+1}(t) E^{\alpha+1}(t)-C E^{\prime}(t), \quad \forall \varepsilon>0, \forall t \geq t_{0} .
\end{aligned}
$$

We then choose $\varepsilon<\frac{m}{C}$ and recall that $\xi^{\prime} \leq 0$ and $E^{\prime} \leq 0$, to get

$$
\begin{aligned}
\left(\xi^{\alpha+1} E^{\alpha} \mathcal{F}\right)^{\prime}(t) & \leq \xi^{\alpha+1}(t) E^{\alpha}(t) \mathcal{F}^{\prime}(t) \\
& \leq-c_{1} \xi^{\alpha+1}(t) E^{\alpha+1}(t)-C E^{\prime}(t), \forall t \geq t_{0}
\end{aligned}
$$

which implies

$$
\left(\xi^{\alpha+1} E^{\alpha} \mathcal{F}+C E\right)^{\prime}(t) \leq-c_{1}^{\alpha+1} \xi(t) E^{\delta+1}(t) .
$$

Let $W=\xi^{\alpha+1} E^{\alpha} \mathcal{F}+C E \sim E$. Then

$$
W^{\prime}(t) \leq-C \xi^{\alpha+1}(t) W^{\alpha+1}(t)=-C \xi^{2 p-1} W^{2 p-1}(t), \forall t \geq t_{0} .
$$

Integrating over $\left(t_{0}, t\right)$ and using the fact that $W \sim E$, we obtain

$$
E(t) \leq C\left[\frac{1}{\int_{t_{0}}^{t} \xi^{2 p-1}(s) d s+1}\right]^{\frac{1}{2 p-2}}, \quad \forall t \geq t_{0}
$$

To establish (4.30) we consider (4.42) and recall Remark 4.4. So, we have

$$
\begin{aligned}
\xi(t) \mathcal{F}^{\prime}(t) & \leq-m \xi(t) E(t)+C \xi(t)(g \circ \nabla u)(t) \\
& =-m \xi(t) E(t)+C \frac{\eta(t)}{\eta(t)} \int_{0}^{t}\left[\xi^{p}(s) g^{p}(s)\right]^{\frac{1}{p}}\|\nabla u(t)-\nabla u(t-s)\|_{2}^{2},
\end{aligned}
$$

where

$$
\eta(t)=\int_{0}^{t}\|\nabla u(t)-\nabla u(t-s)\|_{2}^{2} d s \leq C \int_{0}^{t}\|\nabla u(t)\|_{2}^{2}+\|\nabla u(t-s)\|_{2}^{2} d s
$$




$$
\begin{aligned}
& \leq C \int_{0}^{t}[E(t)+E(t-s)] d s \leq 2 C \int_{0}^{t} E(t-s) d s \\
& =2 C \int_{0}^{t} E(s) d s<2 C \int_{0}^{+\infty} E(s) d s<+\infty .
\end{aligned}
$$

Applying Jensens's inequality (2.15) for the second term on the right hand side of (4.46), with $G(y)=$ $y^{\frac{1}{p}}, y>0, f(s)=\xi^{p}(s) g^{p}(s)$ and $h(s)=\|\nabla u(t)-\nabla u(t-s)\|_{2}^{2}$, to get

$$
\xi(t) \mathcal{F}^{\prime}(t) \leq-m \xi(t) E(t)+C \eta(t)\left[\frac{1}{\eta(t)} \int_{0}^{t} \xi^{p}(s) g^{p}(s)\|\nabla u(t)-\nabla u(t-s)\|_{2}^{2} d s\right]^{\frac{1}{p}},
$$

where we assume that $\eta(t)>0$, otherwise we get $\|\nabla u(t)-\nabla u(t-s)\|=0$ and hence from (4.41) we have

$$
E(t) \leq C e^{-m t} .
$$

Therefore, we obtain

$$
\begin{aligned}
\xi(t) \mathcal{F}^{\prime}(t) & \leq-m \xi(t) E(t)+C \eta^{\frac{p-1}{p}}(t)\left[\xi^{p-1}(0) \int_{0}^{t} \xi(s) g^{p}(s)\|\nabla u(t)-\nabla u(t-s)\|_{2}^{2} d s\right]^{\frac{1}{p}} \\
& \leq-m \xi(t) E(t)+C\left(-g^{\prime} \circ \nabla u\right)^{\frac{1}{p}}(t) \leq-m \xi(t) E(t)+C\left(-E^{\prime}(t)\right)^{\frac{1}{p}} .
\end{aligned}
$$

Multiplying by $\xi^{\alpha}(t) E^{\alpha}(t)$, for $\alpha=\rho-1$, and repeating the same computations as in above, we arrive at

$$
E(t) \leq K\left[\frac{1}{1+\int_{t_{0}}^{t} \xi^{p}(s) d s}\right]^{\frac{1}{p-1}}, \quad \forall t \geq t_{0}
$$

This completes the proof of our main result.

The following examples illustrate our result given by Theorem 4.4.

Example 1. Let $g(t)=\frac{a}{(1+t)^{t}}, \nu>2$, where $a>0$ is a constant so that $\int_{0}^{+\infty} g(t) d t<1$. We have

$$
g^{\prime}(t)=-\frac{a \nu}{(1+t)^{\nu+1}}=-b\left(\frac{a}{(1+t)^{\nu}}\right)^{\frac{\nu+1}{\nu}}=-b g^{p}(t), \quad p=\frac{\nu+1}{\nu}<\frac{3}{2}, \quad b>0 .
$$

Therefore (4.29), with $\xi(t)=b$, yields $\int_{0}^{+\infty}\left(\frac{1}{b^{2 p-1} t+1}\right)^{\frac{1}{2 p-2}} d t<\infty$ and hence by (4.30) we get

$$
E(t) \leq \frac{C}{(1+t)^{\frac{1}{p-1}}}=\frac{C}{(1+t)^{\nu}}
$$

which is the optimal decay obtained in [7]. 
Example 2. Let $g(t)=a e^{-(1+t)^{\nu}}, 0<\nu \leq 1$ where $0<a<1$ is chosen so that $\int_{0}^{+\infty} g(t) d t<1$. Then

$$
g^{\prime}(t)=-a \nu(1+t)^{\nu-1} e^{-(1+t)^{\nu}}=-\xi(t) g(t)
$$

where $\xi(t)=\nu(1+t)^{\nu-1}$ which is a decreasing function and $\xi(0)>0$. Therefore we can use (4.27) to deduce

$$
E(t) \leq C e^{-\lambda(1+t)^{\nu}}
$$

\section{References}

[1] Alabau-Boussouira. F.; Convexity and weighted integral inequalities for energy decay rates of nonlinear dissipative hyperbolic systems, Applied Mathematics and Optimization, vol. 51, no. 1, (2005), 61-105.

[2] Berrimi. S.; Messaoudi. S.A.; Exponential decay of solutions to a viscoelastic equation with nonlinear localized damping, Electronic Journal of Differential Equations, vol. 88, (2004), 1-10.

[3] Berrimi. S.; Messaoudi. S.A.; Existence and decay of solutions of a viscoelastic equation with a nonlinear source, Nonlinear Analysis: Theory, Methods \& Applications, vol. 64, no. 10, (2006), pp. 2314-2331.

[4] Burns. J.A.; Liu. Y. Z.; Zheng. S.; On the energy decay of a linear thermoelastic bar, J. Math. Anal. Appl. 179, (1993), 574-591.

[5] Cavalcanti. M.M.; Domingos Cavalcanti. V. N.; Prates Filho. J.S.; Soriano. J.A.; Existence and uniform decay rates for viscoelastic problems with nonlinear boundary damping, Differential and Integral Equations, vol. 14, no. 1, (2001), $85-116$.

[6] Cavalcanti. M.M.; Domingos Cavalcanti. V. N.; Soriano. J.A.; Exponential decay for the solution of semilinear viscoelastic wave equations with localized damping, Electronic Journal of Differential Equations, vol. 44, (2002), 1-14.

[7] Cavalcanti. M.M.; Oquendo. H. P.; Frictional versus viscoelastic damping in a semilinear wave equation, SIAM Journal on Control and Optimization, vol. 42, no. 4, (2003), pp. 1310-1324.

[8] Cavalcanti. M.M.; Domingos Cavalcanti. V.N.; Martinez. P.; General decay rate estimates for viscoelastic dissipative systems, Nonlinear Analysis: Theory, Methods \& Applications, vol. 68, no. 1, (2008), 177-193.

[9] Li. F.; Zhao. N.; Uniform energy decay rates for nonlinear viscoelastic wave equation with nonlocal boundary damping, Nonlinear Analysis: Theory, Methods \& Applications, vol. 74, no. 11, (2011), 3468-3477.

[10] Li. F.; Zhao. Z.; Chen. Y.; Global existence uniqueness and decay estimates for nonlinear viscoelastic wave equation with boundary dissipation, Nonlinear Analysis: Real World Applications, vol. 12, no. 3, (2011), 1759-1773.

[11] Lu. L.; Li. S.; Chai. S.; On a viscoelastic equation with nonlinear boundary damping and source terms: global existence and decay of the solution, Nonlinear Analysis: Real World Applications, vol. 12, no. 1, (2011), 295-303.

[12] Messaoudi. S. A.; General decay of the solution energy in a viscoelastic equation with a nonlinear source, Nonlinear Analysis: Theory, Methods \& Applications, vol. 69, no. 8, (2008), pp. 2589-2598.

[13] Messaoudi. S.A; Mustafa. M.I.; On convexity for energy decay rates of a viscoelastic equation with boundary feedback, Nonlinear Analysis: Theory, Methods \& Applications, vol. 72, no. 9-10, (2010), 3602-3611.

[14] Messaoudi. S.A.; Waled Al-Khulaifi; General and optimal decay for a quasilinear viscoelastic equation, Applied Mathematics Letters. vol. 66, (2017), 16-22.

[15] Muňoz Rivera. J.E; Peres Salvatierra A.; Asymptotic behaviour of the energy in partially viscoelastic materials, Quarterly of Applied Mathematics, vol. 59, no. 3 (2001), pp. 557-578.

[16] Park. J.Y.; Park. S.H.; Existence and asymptotic stability for viscoelastic problems with nonlocal boundary dissipation, Czechoslovak Mathematical Journal, vol. 56, no. 2, (2006), 273-286. 
[17] Shun-Tang. W.; Hsueh-Fang Chen.; Uniform decay of solutions for a nonlinear viscoelastic wave equation with boundary dissipation, Journal of Function Spaczs and Applications, (2012), vol. Article ID 421847.

[18] Vitillaro. E.; Global existence for the wave equation with nonlinear boundary damping and source terms, Journal of Differential Equations, vol. 186, no. 1 (2002), pp. 259-298. 